ARTÍCULOS DE INVESTIGACIÓN

\title{
Los abogados graduados en la Universidad Metropolitana de Venezuela (2006-2016). Estudio de una cohorte profesional en tiempos de revolución
}

\author{
Os advogados graduados na Universidade Metropolitana de Venezuela (2006-2016). \\ Estudos de um coorte profissional em tempos de revolução
}

Lawyers graduated at the Universidad Metropolitana of Venezuela (2006-2016). Study of a professional cohort in times of revolution

\author{
Victoria Capriles iD \\ Universidad Metropolitana, Venezuela \\ Rogelio Pérez-Perdomo \\ Universidad Metropolitana, Venezuela
}

\begin{abstract}
RESUMEN Este artículo explora la trayectoria profesional de los egresados en Derecho de la Universidad Metropolitana de Caracas entre los años 2006 y 2016. Más de la mitad de los 353 egresados localizados trabajan fuera de Venezuela con una alta proporción de personas en puestos relacionados con la operación del sistema jurídico. Este hallazgo va contra la opinión común de que la educación jurídica venezolana prepara para trabajar solo en el ámbito nacional. Las entrevistas exploraron la opinión de los graduados sobre su educación jurídica y las estrategias de adaptación a otras culturas jurídicas.
\end{abstract}

PALABRAS CLAVE Abogados de Venezuela, educación jurídica, abogados expatriados, crisis venezolana.

RESUMO Este artigo explora a carreira profissional de graduados em Direito da Universidade Metropolitana de Caracas de 2006 a 2016. Mais da metade dos 353 graduados localizados trabalham fora da Venezuela com uma alta proporção de pessoas em empregos relacionados. com o funcionamento do sistema jurídicol. Essa conclusão contraria o senso comum de que a educação jurídica venezuelana se prepara para trabalhar apenas em nível nacional. As entrevistas exploraram a opinião dos formandos sobre sua formação jurídica e estratégias para se adaptarem a outras culturas jurídicas. 
PALAVRAS-CHAVE Advogados da Venezuela, educação jurídica, advogados expatriados, crise venezoelana.

\begin{abstract}
This article explores the professional career of law graduates from the Universidad Metropolitana in Caracas from 2006 to 2016. More than half of the 353 located graduates work outside of Venezuela, with a sizable proportion in law-related jobs. This finding goes against the common view that Venezuelan legal education is only nationally focused. The interviews explored the graduates' opinions regarding their legal education and their strategies for adapting to foreign legal cultures.
\end{abstract}

KEYWORDS Venezuelan lawyers, legal education, expatriate lawyers, Venezuelan crisis.

\title{
Estudios jurídicos en Venezuela y en la Universidad Metropolitana
}

La Universidad Metropolitana (Unimet) tenía ya treinta años cuando se creó la Escuela de Derecho en 2002. La universidad fue creada por empresarios importantes que apoyaban una modernización de la educación universitaria y comenzó a funcionar con facultades de ingeniería, economía, y ciencias y artes. Ofreció carreras en diferentes ramas de ingeniería, matemáticas, administración, economía, educación preescolar e idiomas modernos. A pesar del carácter pragmático de las carreras ofrecidas, la universidad se preocupó de ofrecer estudios generales con un fuerte componente humanístico y desarrollar una biblioteca cuya base inicial fue la biblioteca personal de Pedro Grases, un humanista y bibliógrafo hispano-venezolano de larga trayectoria.

Cuando comenzó el siglo XXI no había ausencia de escuelas de derecho en Venezuela ni en Caracas. En 2001 había un total de 16 escuelas de derecho en el país. Cuatro correspondían a universidades estatales autónomas, que conforme a la tradición venezolana son gratuitas para los estudiantes, y doce a universidades privadas. En Caracas había cinco escuelas de derecho. La más antigua es parte de la Universidad Central de Venezuela existente desde 1720 y era en 2001 la única perteneciente a una universidad estatal autónoma. Según los boletines estadísticos de la Oficina de Planificación del Sector Universitario (OPSU), contaba con 2.999 estudiantes. Estaban además las escuelas de la Universidad Católica Andrés Bello (1.778 estudiantes) y la Universidad Santa María (5.054 estudiantes), ambas creadas en la década de 1950, cuando se permitieron universidades privadas en Venezuela. Había dos escuelas recientes correspondientes a las universidades José María Vargas (639 estudiantes) y Monteávila (56 estudiantes). En total la población estudiantil en derecho era de 39.116 estudiantes en 2001. De ellos el 27\% estudiaban en Caracas y de estos la mitad en la Universidad Santa María (Pérez-Perdomo, 2006).

Este mismo período ha sido de cambios políticos muy radicales. Hasta 1999 Ve- 
nezuela era una democracia representativa en la cual los partidos políticos jugaban un papel muy importante. La judicatura estaba muy penetrada por los partidos y, en general, el estado de derecho era débil. La revolución iniciada en 1999 rápidamente planteó un proyecto socialista y se consolidó como un gobierno autoritario con un férreo control sobre los jueces y las demás instituciones del Estado. Las apariencias democráticas, que sobrevivieron durante la presidencia de Hugo Chávez, desaparecieron bajo Nicolás Maduro, quién asumió el poder en 2013, y se ha caracterizado por ser un presidente con muy baja popularidad y con una acentuada tendencia represiva. El débil estado de derecho no tuvo ninguna capacidad de resistencia frente a los embates de la revolución. La revolución chavista siguió políticas económicas socialistas, o más bien antiempresariales, que desembocó en una severa crisis de escasez e hiperinflación. Probablemente más del 10\% de la población venezolana ha emigrado.

El régimen chavista propició la creación de escuelas de derecho en universidades estatales no autónomas (las cuales se caracterizan por poseer una fuerte corriente ideológica acorde al gobierno actual y una enorme injerencia del poder político). De esta manera, para 2015 había 26 escuelas de derecho. Lamentablemente no hay datos sobre el número de estudiantes de derecho porque la OPSU ha dejado de publicar estadísticas y probablemente tampoco compila datos. ${ }^{1}$ Sin duda, el proyecto más importante del régimen es la Universidad Bolivariana (UBV), que ha municipalizado los estudios jurídicos. Esto quiere decir que hay estudios de derecho en todas las ciudades del país, incluyendo ciudades muy pequeñas, bajo el paraguas de la Universidad Bolivariana. Ni la UBV ni el gobierno publican datos sobre el número de estudiantes o de graduados en derecho, pero veremos luego que esta universidad se ha convertido en la mayor productora de abogados del país.

Lo característico del panorama actual de los estudios jurídicos es la variedad de los proyectos educativos (Martínez Meucci, 2013; Pinilla-Rodríguez, Reinoso-Vásquez y López-Merino, 2017; Carrington, 2017; González Galván, 2013; Castellanos Claramunt, 2019; Castro y Oliveira, 2018; Pérez Perdomo, 2016). Hay facultades de derecho que están básicamente dedicadas a la actividad docente y contratan profesores solo por las horas de docencia, mientras que otras facultades contratan profesores de tiempo completo como parte de su personal, y esperan que un número de sus profesores hagan investigación y publiquen. Hay facultades de derecho que utilizan fundamentalmente el método tradicional de la clase magistral para explicar las distintas ramas del derecho, mientras otras prestan también atención a las competencias que tratan de comunicar a los estudiantes y utilizan problemas y casos para la discusión. Hay facultades que privilegian ciertas ramas del derecho, como el derecho penal o el constitucional, mientras otras se enfocan en la actividad de negocios. En la UBV no

1. En 2015 Rogelio Pérez-Perdomo visitó en varias ocasiones la sede de la OPSU y conversó con varios funcionarios, lo que lo llevó a la conclusión de que no se dispone de los datos. 
se estudian las ramas tradicionales del derecho, sino que se trata de formar personas que puedan promover las ideas socialistas-chavistas y operar como funcionarios del Estado bajo el presente régimen. ${ }^{2}$

La Universidad Metropolitana se esfuerza en tener profesores de tiempo completo, en promover la investigación, en usar problemas y casos en la educación, en atender a las competencias que se espera adquieran los estudiantes, entre ellas que se expresen adecuadamente por escrito y oralmente. Los estudiantes deben desarrollar proyectos de emprendimiento, llegar a un elevado nivel de inglés, hacer pasantías profesionales, y realizar investigaciones que terminan en un trabajo de grado. Estas son políticas generales de la universidad que se aplican también en los estudios jurídicos. Además, su Escuela de Derecho requiere un examen comprehensivo, en el cual se examina si los estudiantes han adquirido las competencias básicas para desempeñarse profesionalmente y pueden utilizar apropiadamente los argumentos jurídicos. ${ }^{3}$ Otro rasgo distintivo de los estudios jurídicos en la Unimet es la posibilidad de que los estudiantes desarrollen conjuntamente dos carreras de estudio. Son así relativamente frecuentes los estudiantes que obtienen dos grados (por ejemplo, en Derecho y Estudios Liberales - un programa interdisciplinario en las áreas de filosofía y ética, política, economía e historia-, o en Derecho y Administración). Todos estos rasgos hacen peculiares los estudios jurídicos en la Universidad Metropolitana en el panorama de la educación jurídica venezolana.

\section{Las profesiones jurídicas}

En Venezuela no disponemos de un censo de abogados. Es importante destacar que en Venezuela son abogados todos los graduados en Derecho y que el título de abogado es otorgado directamente por las universidades. Tenemos las cifras de un seguro obligatorio que los abogados deben suscribir como requisito de acceso para cualquiera de los tribunales del país. Este seguro, denominado Inpreabogado (acrónimo para Instituto de Previsión Social del Abogado), suministra los datos de los inscritos por año y por universidad a quienes lo soliciten. Esta información permite calcular el tamaño de la profesión jurídica, pero no permite clasificarla por sexo ni conocer la jurisdicción donde se desempeñan.

El cuadro 1 ofrece los datos agregados de los nuevos inscritos en el Inpreabogado

2. Esto fue corroborado por Rogelio Pérez-Perdomo en 2013, cuando tuvo acceso al pensum de la carrera de Derecho de la UBV. Observó que no había ninguna asignatura «tradicional» como Derecho Mercantil, Penal o Administrativo, pero había asignaturas tituladas «Regulación jurídica de las relaciones privadas» $\mathrm{y}$ «Economía social y desarrollo endógeno sustentable». La UBV tiene convenios con tribunales penales municipales y otros entes, y sus egresados pasan directamente a ser funcionarios públicos.

3. Para mayor información, véase http://www.unimet.edu.ve/derecho/. 
Cuadro 1. Nuevos graduados en Derecho en el período 1990-2016.

\begin{tabular}{|lcc|}
\hline Período & Nuevos graduados registrados & Promedio por año \\
\hline $1990-1999$ & 38.043 & 3.804 \\
\hline $2000-2009$ & 65.713 & 6.573 \\
\hline $2010-2016$ & 124.122 & 20.687 \\
\hline Total 1990-2016 & 227.878 & 11.143 \\
\hline
\end{tabular}

Fuente: Inpreabogado.

Cuadro 2. Graduados en Derecho de la Unimet por año.

\begin{tabular}{|cc|}
\hline Año & Graduados en derecho de la Unimet \\
\hline 2006 & 17 \\
2007 & 46 \\
2008 & 32 \\
\hline 2009 & 53 \\
2010 & 26 \\
2011 & 74 \\
2012 & 48 \\
2013 & 55 \\
2014 & 56 \\
2015 & 39 \\
2016 & 29 \\
2017 & 33 \\
\hline
\end{tabular}

Fuente: Secretaría General de la Unimet.

para los últimos 27 años. Como puede apreciarse, en ese período se han inscrito más de 227.878 abogados, de los cuales 47.199 se han graduado en la Universidad Bolivariana: esta universidad aparece como el mayor contribuyente $(21 \%)$ al rápido incremento de los abogados en Venezuela.

Entre 2006 y 2017 la Universidad Metropolitana ha graduado 508 abogados, el $0,25 \%$ de todos los nuevos graduados (cuadro 2).

La contribución de la Universidad Metropolitana es cuantitativamente insignificante, pero es probable que la contribución cualitativa tenga relevancia. Esto requerirá que analicemos qué hacen y dónde están los graduados en esta universidad.

La atención a los cambios de la profesión en los últimos 26 años no permite apreciar los cambios muy sustanciales de la sociedad venezolana en la segunda mitad del siglo XX y en lo que va del siglo XXI. Venezuela fue un país de muy rápido crecimiento económico desde 1950 hasta 1980, y desde 1958 desarrolló un sistema democrático. Numerosas empresas se establecieron en Venezuela, lo que produjo una rápida industrialización. Un enorme flujo migratorio, primero de europeos, luego de otros países latinoamericanos, y finalmente del Medio y Próximo Oriente, dio 
un aire cosmopolita a Caracas y las principales ciudades venezolanas. Las profesiones, incluyendo la de abogado, se desarrollaron igualmente (Pérez-Perdomo, 2003: 414-478). En Caracas se consolidaron firmas modernas de abogados primero que en otros países de América Latina. Baker \& McKenzie estableció en Caracas la primera firma fuera de los Estados Unidos en la década de 1950. Abogados norteamericanos, europeos y de otros países latinoamericanos se incorporaron a firmas de abogados produciendo una rápida internacionalización de la abogacía de negocios (Mészáros y Pérez-Perdomo, 2017). A la vez eran numerosos los abogados venezolanos que hacían posgrados en el exterior, contribuyendo a esa internacionalización (Gómez y Pérez-Perdomo, 2018). La llamada 'apertura petrolera' de la década de los noventa produjo un verdadero boom en el crecimiento de las firmas de abogados de negocios. Paralelamente, las consultorías jurídicas de las empresas crecieron en tamaño e importancia (Pérez-Perdomo, 2001: 301-324).

La revolución iniciada por Hugo Chávez se declaró antiimperialista y anticapitalista. Nacionalizó toda clase de industrias y aun de empresas comerciales tanto en manos de inversionistas extranjeros como locales. Esto produjo una abrupta detención del crecimiento económico y de las firmas de abogados. El cuadro 3 muestra cómo las firmas han sido afectadas de distinta manera, pero en promedio ha significado una disminución de su tamaño.

Nótese que la firma Hoet, Peláez, Castillo \& Duque se denominaba previamente Bentata, Hoet \& Asociados, así como la firma Norton Rose Fullbright se denominaba Macleod Dixon. Esta última, para el año 1999 era nueva en el mercado. En 2009 alcanzó a tener 48 abogados.

El régimen chavista desde muy temprano tomó el control del sistema de justicia y se caracterizó por la violación del derecho de propiedad. En opinión de Canova, Herrera, Rodríguez y Graterol (2014), la judicatura fue desprofesionalizada y se ha puesto «al servicio de la revolución», situación descrita también por otros autores (Chavero, 2011; Louza, 2011; Pérez-Perdomo, 2011). El problema no concierne solo el ámbito judicial. Los abogados se quejan del trato arbitrario y desconsiderado que reciben en la administración pública y en notarías y registros. La violación de los derechos humanos se convirtió en un rasgo que frecuentemente ha provocado la condena del país en los foros internacionales. Las reiteradas condenas en la Corte Interamericana de Derechos Humanos llevaron al Gobierno a la renuncia del Tratado Interamericano de Derechos Humanos en 2012 (Pérez-Perdomo y Santacruz, 2017).

Las políticas del gobierno han llevado a graves trastornos en la sociedad venezolana. La delincuencia violenta creció desmesuradamente, en parte promovida por el Gobierno que ha protegido y armado a grupos paramilitares supuestamente como preparativo para resistir una invasión de los Estados Unidos (Briceño-León y Camardiel, 2015). La represión se ha hecho severa: centenares de jóvenes han sido asesinados en manifestaciones públicas y millares han sido sometidos a juicios penales, 
Cuadro 3. Cambio en el número de abogados de las principales firmas en el período 1999-2017

\begin{tabular}{|lcc|}
\hline Nombre de la firma (año de fundación) & $\mathbf{1 9 9 9}$ & $\mathbf{2 0 1 7}$ \\
\hline Hoet, Peláez, Castillo \& Duque (1942) & 59 & 56 \\
\hline Baker \& McKenzie (1955) & 76 & 49 \\
\hline D'Empaire Reyna (1972) & 35 & 44 \\
\hline Travieso Evans Arria Rengel \& Paz (1920) & 45 & 38 \\
\hline Mendoza Palacios Acedo Borjas (1945) & 34 & 34 \\
Rodríguez \& Mendoza (1910) & 44 & 33 \\
Araque Reyna Sosa \& Brito (1986) & 25 & 33 \\
Norton Rose Fullbright (1997) & 12 & 31 \\
\hline Tinoco Travieso Planchart \& Núñez (1914) & 44 & 22 \\
Torres Plaz \& Araujo (1972) & 33 & 22 \\
\hline Promedio & 40,6 & 36,2 \\
\hline
\end{tabular}

Fuentes: Gómez \& Pérez Perdomo (2018) y Latin Lawyer 250, 2017.

incluso ante tribunales militares (Pérez-Perdomo y Santacruz, 2017: 11-34). No es una sorpresa que esta situación haya producido una emigración creciente de venezolanos. ${ }^{4}$ Los primeros en emigrar fueron los profesionales y también quienes habían inmigrado en la segunda mitad del siglo XX y sus hijos. La emigración es hoy en día mucho más general.

El estimado de número de emigrantes varía. La Organización Mundial para las Migraciones ofrece la cifra de 1,6 millones, pero esto no incluye a las personas con otra nacionalidad, grupo que concentra una cifra muy importante de migrantes. ${ }^{5}$ Quienes han calculado el número sobre encuestas de hogares cifran en 4.5 millones el número de emigrantes. En todo caso, la cifra parece superar largamente al 10\% de la población venezolana (unos 31 millones). Un estudio de opinión encontró que el $40 \%$ de los venezolanos expresa deseos de emigrar. ${ }^{6}$ La emigración se nota en la cantidad de viviendas vacías en Caracas y las principales ciudades, y la obvia mejoría del congestionamiento automotor. De acuerdo con el Alto Comisionado de las Naciones Unidas para los Refugiados, el «éxodo de venezolanos es uno de los movimientos de población masivos más grandes de América Latina en la historia», generando crisis

4. Cf. «Crisis de Venezuela: 'El éxodo de los venezolanos es el mayor de Latinoamérica en los últimos 50 años'», $B B C, 24$ de agosto de 2018, disponible en https://www.bbc.com/mundo/ noticias-america-latina-45291398.

5. Naiara Galarraga, «Radiografía del gran éxodo venezolano», El País, 26 de marzo de 2018, disponible en http://bit.ly/2MOd25v

6. International Crisis Group, Cómo detener la onda expansiva de la crisis venezolana. Informe sobre América Latina, 65. Bruselas: International Crisis Group, 2018. Disponible en http://bit.ly/2KOw6xN. 
humanitarias importantes en el estado brasilero de Roraima, la ciudad colombiana de Cúcuta y las provincias ecuatorianas de Carchi, Pichincha y El Oro. ${ }^{7}$

Estas circunstancias, unidas a la disminución de asuntos lucrativos en la profesión, contribuyen al interés de los abogados por emigrar. ${ }^{8}$ Un trabajo de investigación en curso ha encontrado un buen número de abogados venezolanos en Europa, Estados Unidos y otros países latinoamericanos, y también en países tan lejanos como Australia, Nueva Zelanda y Myanmar. ${ }^{9}$

En la próxima sección se describe dónde están y qué hacen los abogados graduados en Derecho de la Universidad Metropolitana, y en la conclusión haremos un balance del desempeño de estos graduados y de su contribución a la profesión jurídica y el país.

\section{¿Dónde están y qué hacen los abogados unimetanos?}

Para este estudio se decidió analizar los graduados entre 2006 -la primera promoción- y 2016. Los egresados en 2017 tienen muy poco tiempo de graduados para analizarlos en su desempeño profesional, especialmente porque es frecuente la realización de estudios de posgrado al año siguiente a su graduación. En total, nuestro universo de investigación comprendió 473 abogados, es decir, el número total de egresados en la universidad hasta 2016, datos que fueron obtenidos por medio de la Secretaría General de la Unimet. El aporte de dicha Secretaría permitió acceder a información sobre nombre y apellido, año de graduación, si cursó alguna otra carrera y dirección de correo electrónico de cada uno de los egresados.

A través de la exploración de varias redes sociales enfocadas en el campo laboral, aunado al envío de correos electrónicos a las direcciones que se encuentran disponibles en la base de datos de la Secretaría, y utilizando una metodología de «bola de nieve» (en la cual se contactaba a un egresado y se le solicitaba que sirviera de "puente» entre los investigadores y el resto de los miembros de su promoción con los que aún tuviese contacto frecuente), se obtuvo información actualizada de 353 abogados, es decir, un $75 \%$ de todos los abogados formados en la Unimet durante el período

7. Alto Comisionado de las Naciones Unidas para los Refugiados, «ACNUR aumenta su respuesta tras la declaratoria de emergencia en Ecuador», ACNUR.org, disponible en http://bit.ly/2Zm7EHZ.

8. Las dificultades para ejercer la profesión no sólo afectan a los abogados. El Instituto de Investigaciones de la Universidad Central de Venezuela se encuentra desarrollando un estudio titulado Diáspora médica venezolana en números, a cargo del médico infectólogo y profesor universitario Julio Castro. De acuerdo con él, el $25 \%$ de los médicos venezolanos han salido del país en busca de condiciones óptimas para poder ejercer su vocación. La investigación será publicada en un futuro próximo.

9. El título provisorio de este trabajo en preparación es Globalization blues: Venezuelan expat lawyers, y está siendo realizado por Manuel Gómez, Gilberto Guerrero y Rogelio Pérez-Perdomo. Tanto ese proyecto como el que se presenta en este trabajo están coordinados. 
2006-2016, lo que conformó la base de datos para esta investigación. La información fue recogida entre octubre de 2017 y mayo de 2018. Luce oportuno destacar que una de las autoras de este trabajo, Victoria Capriles, es una abogada egresada de la Unimet, por lo que, en consecuencia, debieron considerarse ciertos elementos de autoetnografía. Sin embargo, considerando la naturaleza de esta investigación, es posible afirmar que las dificultades usuales de la autoetnografía no se presentaron.

Para el análisis, los abogados se separaron en dos grandes grupos: quienes se graduaron entre los años 2006 y 2011, y quienes lo hicieron entre los años 2012 al 2016. Esta distribución se hace porque la variable «años de graduado» se considera importante para la experiencia laboral y, eventualmente, para la apreciación que tengan los graduados de la educación recibida. 191 personas (54\%) se graduaron en el primer grupo y 162 en el segundo (46\%). El promedio de graduado por año es 32 . También para el análisis se distinguió entre hombres y mujeres, pues las oportunidades laborales y la satisfacción con el trabajo pueden ser sensibles al género. Las mujeres predominan entre los graduados (61 a 39). En el primer grupo la proporción es 56 a 44. En el segundo es más marcada (66 a 34).

Otra distinción importante fue por el lugar de residencia. Se distinguió entre quienes viven en Venezuela y quienes están en el extranjero. Estos últimos son mayoría (57\%). Entre los graduados entre 2006 y 2011, la proporción es ligeramente mayor (60\%). Los países más atractivos han sido los Estados Unidos (34\%) y España (22\%). Europa - sin contar España- ha atraído al $33 \% .^{10} \mathrm{El} 30 \%$ restante es residente en países latinoamericanos, pero ningún país en particular concentra una proporción alta. ${ }^{11}$ Solo un pequeño número $(3 \%)$ se encuentra en otras partes del mundo. ${ }^{12}$

\section{Actividad profesional y satisfacción con los estudios}

Entre los abogados residentes en el extranjero, el grupo más antiguo se distribuye equitativamente entre quienes trabajan en el ámbito jurídico ${ }^{13}(48,2 \%)$ y quienes lo hacen en otro ámbito ${ }^{14}(51,8 \%)$. Entre los abogados del grupo más reciente es aun

10. En orden decreciente: Francia, Holanda, Bélgica, Suiza, Italia, Alemania, Reino Unido, Austria y Portugal.

11. En orden decreciente: Panamá, Colombia, México, Perú, Argentina, Guatemala, Ecuador, Costa Rica, Chile, República Dominicana, Curazao y El Salvador.

12. En orden decreciente: Nueva Zelanda, Australia, Emiratos Árabes Unidos e Israel.

13. Se entiende como «ámbito jurídico» tanto estudios como ocupaciones laborales que estén relacionadas con el derecho. Por ejemplo: socios y asociados en firmas de abogados, profesores universitarios de la carrera de Derecho, compliance counsels, immigration case managers, estudiantes de máster o doctorado.

14. Se entiende como «otro ámbito» tanto estudios como ocupaciones laborales que no estén relacionadas con el derecho. Por ejemplo: cargos gerenciales, analistas de negocios, realtors, CEO, estudiantes de escuelas de negocios, art directors, emprendedores, etcétera. 
mayor la proporción que está en el ámbito jurídico (66,3\%), aunque el peso de quienes estudian posgrado es muy significativo. En el grupo de abogados residenciados en Venezuela, los más antiguos tienden a permanecer en el ámbito jurídico $(70,9 \%)$ y esta proporción es algo mayor $(72,2 \%)$ en el grupo de los abogados graduados más recientemente.

Para conocer el grado de satisfacción con las actividades realizadas en la actualidad y su percepción sobre si su educación en la Unimet ha sido de ayuda para alcanzar lo logrado, se distribuyó un cuestionario con un conjunto de preguntas sencillas sobre estos tópicos. Se prepararon dos tipos de cuestionarios: uno para los abogados que trabajan en Venezuela y otro para los residentes en el extranjero. Los cuestionarios se distribuyeron por correo electrónico, pero dadas las dificultades de obtener las direcciones electrónicas no sabemos cuántos lo recibieron. No hubo recursos para hacer un seguimiento telefónico. Obtuvimos cuarenta respuestas.

Quienes se encuentran en el exterior atribuyeron la decisión de emigrar a la situación del país. Mencionaron el colapso económico, el pésimo funcionamiento del sistema jurídico y la violencia delincuencial como factores importantes en su decisión. Esta situación también es resentida por quienes se quedaron. El 25\% de ellos expresó que tienen planes para emigrar en el corto plazo. Encontrarse en el extranjero no se percibe como una situación permanente. $\mathrm{El} 70 \%$ de quienes se encuentran en el extranjero manifestó su deseo de regresar a Venezuela si la situación cambia.

Quienes respondieron al cuestionario expresaron opiniones muy positivas respecto a la educación jurídica que recibieron en la Unimet. Aprecian en particular una educación interdisciplinaria, que estimula la investigación y el pensamiento propio, y que usa casos, problemas y la discusión en clase. El $29 \%$ de quienes respondieron realizaron el programa combinado de derecho y estudios liberales. Consideran que la educación recibida en la Unimet es el elemento diferenciador que los ha distinguido.

Como se prometió confidencialidad, las respuestas que se citan se identifican solo con el año de graduación, con un número referido a ese año, y con el sexo del respondiente. Por ejemplo, «2010, 2, F» indica que se trata de una mujer graduada en 2010 y que el cuestionario fue el segundo recibido entre los graduados de ese año.

Se debe tener en cuenta que, como hemos dicho, solo recibimos cuarenta respuestas y que puede ser una muestra sesgada, en el sentido que quienes no respondieron pueden ser personas que han tenido menos éxito personal o estén menos satisfechos de la educación recibida. También debe ser tenido en cuenta que la tendencia ha sido la realización de estudios de posgrado frecuentemente en el extranjero y que tales estudios adicionales pueden haber sido muy importantes para su inserción en el mercado y la satisfacción con su actividad.

Una egresada del 2013, que ahora vive en España y ha realizado estudios de posgrado en Italia y Hong Kong, comentó: 
Considero que la educación que recibí en Venezuela es una de las mejores del mundo, y su calidad está a nivel de las grandes universidades. Para mí el elemento diferenciador de la educación que recibí es el equilibrio entre la teoría y la práctica, la forma en que nos enseñan a desarrollar un pensamiento analítico, crítico y funcional $(2013,1, F$.)

Otro abogado, que se encuentra trabajando en una prestigiosa firma en Costa Rica, señaló:

El enfoque que tiene la Unimet a la hora de fomentar el autoaprendizaje me resultó fundamental a la hora de asumir el reto de ejercer mi profesión en el extranjero y adaptarme a todo un ordenamiento jurídico nuevo. De muy poco me hubiese servido los modelos de enseñanza que se basan en el estudio pormenorizado y memorización de normas, sin analizar la razón de ser de las mismas $(2016,3, M$.)

Finalmente, un egresado de las primeras promociones, socio en una importante firma de abogados en Venezuela, manifestó:

La formación integral y orientada hacia los negocios que brinda la Unimet fue clave para que haya encontrado carrera en el derecho tributario, la cual es un área que requiere que el abogado entienda a profundidad los negocios y tenga formación en otras áreas (contabilidad, por ejemplo). La experiencia de compartir clases con alumnos de otras carreras y tener materias no vinculadas al derecho me ha permitido ser un profesional más completo, práctico y adaptable a las circunstancias que me han tocado en mi carrera $(2007,1, M$.)

En el grupo de quienes no ejercen una ocupación directamente jurídica, la gran mayoría expresó que su educación fue útil para la actividad que realizan. Varios tenían otra carrera de pregrado, algo facilitado por los planes de estudio de la Universidad Metropolitana. Por ejemplo, una egresada con doble titulación (derecho y estudios liberales), residente en Estados Unidos y con una posición importante en el Banco Interamericano de Desarrollo, sostuvo que:

[La educación en derecho] fue una buena base para entrar al posgrado y trabajos que quería. [Es] importante destacar que nunca entré a la carrera con interés de ser litigante, sino de conocer el derecho como marco referencial para otros proyectos. Por ejemplo, he dirigido proyectos de acceso a la justicia para comunidades rurales/ indígenas en Panamá (2012, 3, F)

Dos de los entrevistados consideraron su educación entre «regular»y «deficiente», teniendo en común el hecho que no se dedican al ámbito jurídico. Uno de estos egresados, quien además posee un título de economía de otra universidad venezolana y trabaja como manager en una firma de consultoría estratégica internacional, sugirió que la Unimet debería enseñar management consulting e investment banking a todos los estudiantes de pregrado. 
Algunos de los egresados de las primeras promociones calificaron su educación como «buena», comentando que notaron una mejoría cuando el plan de estudios fue trimestralizado. Originalmente el plan de estudio de la carrera de Derecho era semestral y contenía un número de asignaturas electivas que permitían al estudiante concentrarse en un área de derecho (estudios jurídicos internacionales, derecho privado y de los negocios, derecho público y regulatorio, derecho procesal y litigio). Esto permitía graduarse con un diplomado en el área escogida si el estudiante aprobaba un número de créditos en esa área. El estudiante podía optar también por asignaturas electivas de la Facultad de Ciencias Económicas y Sociales y, de hecho, muchos optaban por el Diplomado de Administración y Finanzas.

La flexibilidad del plan de estudios - una novedad en Venezuela - causaba confusión entre los estudiantes y administradores. La información en asignaturas propiamente jurídicas era básica, aunque con posibilidad de concentración en un área del derecho. No pocos percibían que tal plan de estudios preparaba solo abogados corporativos, sobre todo porque la mayor parte de los estudiantes optaban por las concentraciones en derecho privado y de los negocios y en administración y finanzas. Cuando la Universidad optó por la trimestralización en septiembre de 2010, se reformó el plan de estudios haciéndolo más rígido y más apegado a la tradición enciclopédica de los estudios jurídicos en Venezuela y el resto de Latinoamérica.

Es importante destacar que la mayor parte de los egresados residentes en Venezuela que se dedican al ámbito jurídico son abogados transaccionales, pues claramente la actividad de litigio ha decaído sustancialmente durante el período en que estos unimetanos han comenzado su actividad profesional. Absolutamente todos los encuestados estuvieron de acuerdo en que el ejercicio en tribunales se ha vuelto sumamente complicado, citando en particular la «corrupción en entes administrativos y tribunales» $(2015,1, M$.), la «burocracia excesiva» $(2016,1, F$.) y la enorme opacidad que rodea al Estado en general, especialmente a la Administración pública, que una abogada describió como «una caja negra» (2009, 1, F.). Además, en un contexto tan politizado como el venezolano, ejercer ciertas áreas del derecho puede acarrear graves consecuencias. ${ }^{15}$ De acuerdo con una abogada egresada en el 2009, radicada en Venezuela y que ahora solo realiza trabajos transaccionales:

Operar con normas jurídicas cuando su fundamento, el ordenamiento constitucional, se encuentra sometido al libre arbitrio de quienes detentan el poder es su-

15. Un abogado, egresado de posgrado en la Unimet, trabajaba como asesor legal de una conocida cadena de supermercados venezolana, la cual en el año 2015 fue acusada de incurrir en irregularidades en la distribución de productos de primera necesidad. Dicho abogado fue llamado a rendir declaraciones a El Helicoide, la sede del SEBIN (policía política del Gobierno) y una vez adentro se le informó de su detención, sin orden judicial. Fue imputado de «boicot» $y$ «desestabilización de la economía» y permaneció nueve meses recluido en El Helicoide. 
mamente difícil desde el punto de vista ético. Esto, aunado a un sentido de riesgo inminente y a un gran temor (quizás cobardía) me ha hecho migrar de las áreas que más me interesan, como el derecho constitucional y administrativo, a otras ramas más pragmáticas, como el derecho corporativo y mercantil (2009, 1, F).

Sin embargo, no todos los egresados se dejan intimidar por el régimen. Un $20 \%$ de todos los abogados unimetanos, tanto en Venezuela como en el exterior, son defensores o activistas de derechos humanos. Varios han realizado pasantías profesionales en la Comisión Interamericana de Derechos Humanos. Una egresada del 2013 se desempeña como abogada en la Fiscalía de la Corte Penal Internacional. Otro egresado del 2011 desarrolla programas sobre VIH y derechos humanos en América Latina, África y Asia a través de una ONG internacional. Dos egresadas (del 2008 y 2016, respectivamente) estuvieron detrás de la denuncia individual presentada recientemente por José Pernalete ante el Comité de Derechos Humanos de la ONU, en contra del Estado venezolano, por la ejecución extrajudicial de su hijo. ${ }^{16}$

Además, el Centro de Derechos Humanos de la Unimet se encuentra dirigido por dos egresadas (del 2008 y 2015, respectivamente). El Centro se encarga de defender penalmente a varios prisioneros políticos, incluido el caso de la Fundación Embajadores Comunitarios ${ }^{17} \mathrm{y}$ a monitorear, rastrear y documentar diversas violaciones de derechos humanos. En junio 2018, la directora ejecutiva del Centro acudió a un debate en el Parlamento Europeo que versó sobre la emergencia humanitaria compleja en Venezuela. De igual forma, otros han seguido una vocación política, algo también complejo en el contexto actual. Elías Sayegh, dirigente opositor y egresado del 2008, fue electo alcalde de un municipio caraqueño en $2017 .^{18}$

Un 38\% de quienes se han enfocado en ámbitos distintos a la operación del sistema jurídico, tanto en Venezuela como en el exterior, cuentan con su propio emprendimiento o están directamente involucrados en actividades en el área económica. La

16. Juan Pablo Pernalete Llovera, becado por excelencia deportiva y estudiante de la Facultad de Ciencias Económicas y Sociales de la Unimet, fue asesinado el 26 de abril de 2017 cuando un Guardia Nacional le disparó una bomba lacrimógena directamente al pecho en medio de una manifestación pacífica.

17. Gregory Hinds, un venezolano-barbadense, es director general de la Fundación Embajadores Comunitarios, una ONG venezolana fundada en 2008 que trabaja con jóvenes en riesgo en áreas marginadas de Venezuela. Su misión es preparar adolescentes en estas áreas para participar en Modelos de las Naciones Unidas. El 31 de enero de 2018 Hinds fue arbitrariamente detenido, y recluido en El Helicoide hasta el 1 de junio de 2018. Fue declarado prisionero de conciencia de Amnistía Internacional, y varias organizaciones, incluyendo Human Rights Watch y la Organización Mundial Contra la Tortura, se pronunciaron a favor de su excarcelación.

18. Su predecesor, David Smolansky, no pudo culminar su período ya que la Sala Constitucional del Tribunal Supremo de Justicia lo inhabilitó políticamente y lo condenó a quince meses de prisión por las protestas antigubernamentales ocurridas en el municipio. Smolansky se encuentra actualmente en el exilio. 
Universidad Metropolitana tiene un marcado enfoque hacia los negocios, que se demuestra en que los estudiantes de todas las carreras deben ver obligatoriamente dos asignaturas enfocadas en desarrollar competencias emprendedoras y la institución cuenta con una incubadora denominada Novos, que ayuda a la concreción de un proyecto de empresa. Es destacable el caso de Juan José Pocaterra, egresado del 2013 (quien también posee un título de Economía de la Unimet) y cofundador de ViKua, empresa dedicada al desarrollo y comercialización de soluciones tecnológicas para la construcción de ciudades inteligentes, reconocida en el 2015 por la revista Forbes México como una de las 30 empresas más prometedoras de América Latina. ${ }^{19}$

Incluso aquellos que han decidido explotar su creatividad han reconocido la importancia de sus estudios jurídicos: Enrique Márquez París, quien se graduó en el 2011, y se ha especializado en el área de la música, actualmente desempeñándose como Rights Clearance Manager, fue receptor del premio Cannes Lion de oro en 2016 por ser el productor del tributo que la cantante pop Lady Gaga dio en honor a David Bowie en los $58^{\text {th }}$ Annual Grammy Awards. Según Márquez París, «lo más importante que pude aprender [en mis estudios de Derecho] y que todavía utilizo ha sido la capacidad de análisis para enfrentar cualquier tipo de situación».

En cuanto al tema de género, aunque no se notó ningún tipo de diferencia a nivel de oportunidades, pues abogados unimetanos de ambos sexos se encuentran en buenos puestos laborales, es notorio el hecho que las abogadas suelen pasar por situaciones que sus colegas hombres pocas veces deben sortear. Por ejemplo, una egresada del 2008 considera que el ser mujer sí influye en el ejercicio de la profesión, al menos en el ámbito del derecho penal: nunca visita un ente policial sin el respaldo de uno de sus socios hombres («por seguridad»), y sabe de al menos una oportunidad en que a una colega suya un funcionario policial le ofreció resolver un problema en relación con un detenido a cambio de favores sexuales. Otra egresada del 2015, que litigaba en derecho laboral, declaró que tuvo que soportar proposiciones inapropiadas por parte de trabajadores tribunalicios y en un par de ocasiones observó a compañeras suyas llegar visiblemente afectadas de los tribunales debido a comentarios obscenos que recibieron de parte de otros abogados. Finalmente, otra egresada del 2015, actualmente residente en Ciudad de México, comentó que en ese país también ha sufrido discriminación debido al género:

En México es un tema real, serio, el despacho en el que estoy ahora se jacta de ser uno de los despachos del país con mayor inclusión a nivel de género y solo el $30 \%$ de los abogados somos mujeres [...] una de las razones por las que renuncié a la firma en la que trabajaba anteriormente fue porque sentía un rechazo real hacia mí por el

19. Mónica Ocampo, «30 promesas de negocios de Centroamérica», Forbes México, 10 de septiembre de 2015, disponible en http://bit.ly/2Kjevyq. 
hecho de ser mujer, de hecho, en el departamento corporativo de la Ciudad de México yo era la única mujer, desde socios hasta lo más bajo, ni siquiera había pasantes mujeres $(2015,5, F)$.

\section{Balance}

Es frecuente oír la opinión que la formación jurídica (Hernández Santiago, 2017; Pazos Padilla y Fabara, 2018) prepara para el ejercicio de una de las profesiones jurídicas (Pérez Hurtado, 2018; Aránguez Sánchez, 2018; Monroy-Cely, 2018) en el ámbito nacional y que no es útil en el extranjero. Es una opinión que no carece de fundamento, pues efectivamente las reglas de derecho tienen un ámbito territorial y los distintos países generalmente exigen que quienes vayan a ejercer el derecho comprueben que conocen el derecho nacional. Los procedimientos de habilitación profesional son frecuentemente exigentes. El hallazgo más importante de este estudio es que haya un número tan alto de abogados graduados en la Unimet (57\%) que se encuentren fuera de Venezuela y aún más notable que la mayoría de este subgrupo (54\%) trabaje en el ámbito jurídico. En cambio, no es enteramente una sorpresa que tres cuartos de quienes se han quedado en Venezuela trabajen en el ámbito jurídico.

De los 353 abogados estudiados, solo una egresada del 2012 se encuentra haciendo un oficio considerado menor, pues trabaja como conserje en Canadá, sin embargo, también se entiende que quienes suelen realizar este tipo de oficios (taxistas, mesoneros, vendedores ambulantes) son menos visibles que aquellos considerados «más exitosos» en el sentido tradicional, por lo que no sería de extrañar que algunos de los egresados que conforman el $25 \%$ del que no se obtuvo información estén trabajando en algún oficio menor.

No conocemos qué porcentaje de graduados en Derecho en universidades venezolanas se encuentran en el extranjero, por lo tanto, no hay cómo confirmar si la Unimet cuenta con un porcentaje más elevado de egresados migrantes que otras universidades, o si por el contrario está dentro del promedio. Lo que sí se puede inferir de la información obtenida es que nuestros egresados parecen ocupar importantes posiciones en empresas y organizaciones internacionales, y habría que buscar una explicación a este fenómeno. Es posible que, al centrarse en la educación por competencias y no en la transmisión de conocimientos sobre las reglas y principios jurídicos que están en el eje de las otras escuelas, se facilita la adaptación a sistemas jurídicos distintos.

Esto es un efecto involuntario de la educación por competencias que no fue pensada para facilitar la emigración, sino para dar flexibilidad a los egresados en una sociedad en cambio acelerado. En segundo lugar, la Unimet ha dado peso al ámbito internacional. Fue la primera universidad de Venezuela en establecer como asignatura obligatoria al derecho comparado y ha tenido asignaturas electivas como 
«Introducción al derecho y la cultura política de los Estados Unidos», y «Derecho y sociedad en América Latina». Durante un tiempo funcionó un Diplomado en Estudios Jurídicos Internacionales. Instrumentalmente se le da importancia al inglés como idioma extranjero base, y quienes hacen el programa combinado de derecho y estudios liberales, deben aprender un segundo idioma extranjero. Tampoco este rasgo fue pensado para facilitar la emigración, sino que se pensó para dar una base cultural más sólida y una educación jurídica más apropiada para una época de globalización.

Aun cuando la adaptación a una cultura jurídica extranjera no es fácil para nadie, es probable que sea algo menos difícil para los egresados de la Unimet que para los egresados de otras escuelas de derecho.

También es probable que los abogados graduados en la Unimet tengan una apertura mayor para desempeñarse en campos distintos al derecho que los graduados en otras escuelas. En las otras escuelas el peso de las asignaturas jurídicas es mucho más importante y prácticamente los estudiantes no estudian nada distinto al derecho. Los estudiantes de la Unimet tienen una formación más variada y un número importante de ellos completa el Diplomado de Administración y Gerencia. En ese sentido, nos ha sorprendido el alto número de abogados Unimet que se quedaron en Venezuela y trabajan en el ámbito jurídico. Los trabajos sobre abogados en Venezuela han realizado estimaciones que al menos un tercio de los abogados venezolanos trabajan en tareas sin relación directa con el derecho, muchos de ellos en la Administración pública. Esta es una proporción que probablemente se ha incrementado sustancialmente en los últimos años. En cambio, solo uno de los 353 graduados unimetanos de los que tenemos noticia trabaja en la Administración pública, un egresado del 2010 que se desempeña como aduanero en el Servicio Nacional Integrado de Administración Aduanera y Tributaria. Sin embargo, no podemos afirmar con seguridad que no existan desempleados u ocupados en tareas menores, pues faltó localizar a un cuarto de todos los graduados.

Los autores están conscientes que esta investigación puede ser considerada preliminar. Estaba fuera de nuestros recursos hacer un esfuerzo para localizar a un número mayor de graduados $\mathrm{y}$, sobre todo, perseguirlos para que respondieran el cuestionario. Recomendaríamos que el estudio sobre dónde están y qué hacen los graduados, lo mismo sobre cómo valoran la formación recibida, sea una tarea institucional a la cual la Universidad Metropolitana dedique atención y recursos. Aparte de tener propósitos académicos y de evaluación de la formación provee la universidad, tal información puede ser muy útil si se piensa que los egresados pueden ser una fuente de apoyo y recursos para la institución, como lo son en otros países. Este estudio carece igualmente de profundidad comparativa porque no hemos encontrado estudios similares realizados en otras universidades venezolanas o latinoamericanas. 
Tal vez la contribución de este estudio es abrir un campo para la investigación sobre la educación jurídica y su relación con la actividad profesional de los abogados en una época especialmente difícil para Venezuela y para las profesiones jurídicas.

\section{Referencias}

Aránguez Sánchez, Tasia (2018). «Ética de la virtud y profesiones jurídicas». Problema Anuario de Filosofía y Teoría del Derecho, (12): 169-197.

Briceño-León, Roberto y Alberto Camardiel (2015). Delito organizado, mercados ilegales y democracia en Venezuela. Caracas: Alfa.

Canova, Antonio, Luis Herrera, Rosa Rodríguez y Giuseppe Graterol (2014). El TSJ al servicio de la revolución. Caracas: Galipán.

Carrington, Paul (2017). «El derecho y el río». Academia. Revista sobre Enseñanza del Derecho de Buenos Aires, 15(30): 15-25.

Castellanos Claramunt, Jorge (2019). «Educación y participación ciudadana: mejorar la docencia universitaria de la mano de los derechos humanos». Revista de Educación y Derecho, 19: 1-21.

Castro, Nazaré y Claudia Oliveira (2018). «Dilemas do ensino jurídico frente a complexidade do momento atual». Revista de Pesquisa e Educação Jurídica, 4(2): 51-68.

Chavero, Rafael (2011). La justicia revolucionaria: una década de reestructuración (o involución) judicial en Venezuela. Caracas: Aequitas.

Gómez, Manuel y Rogelio Pérez-Perdomo (2018). Big law in Venezuela: from globalization to revolution. Londres: Palgrave Macmillan.

GonZÁlez Galván, Jorge (2013). «Educación jurídica, investigación y derechos humanos inteligentes». Boletín Mexicano de Derecho Comparado, 46(137): 499-527.

Hernández Santiago, Pedro (2017). «Breve diagnóstico sobre la oferta académica para la formación de los profesionales del Derecho en México». Revista de la Educación Superior, 46(181): 55-74.

LouzA, Laura (2011). La revolución judicial en Venezuela. Caracas: Fundación Estudios de Derecho Administrativo.

Martínez Meucci, Miguel Ángel (2013). «La revolución iliberal venezolana y su política exterior». Análisis Político, 26(77): 211-231.

Mészáros, Maritza y Rogelio Pérez-Perdomo (2017). La internacionalización de los abogados de negocios en Venezuela. Caracas: Universidad Metropolitana.

Monroy-Cely, Daniel (2018). «Hacia una educación jurídica práctica y globalizada en Colombia». Revista Iberoamericana de Educación Superior, 9(26): 130-152.

Pazos Padilla, Rina y Jorge Fabara (2018). «La carrera de Derecho en Ecuador: Análisis de los planes de estudio en el 2018». Revista Pedagogía Universitaria y Didáctica del Derecho, 5(2): 9-30. 
Pérez Hurtado, Luis Fernando (2018). «El sistema de enseñanza del derecho y acceso a las profesiones jurídicas en Alemania: Lecciones para el debate en México». Boletín Mexicano de Derecho Comparado, 51(151): 263-311.

Pérez Perdomo, Rogelio y Andrea Santacruz (2017). "The Chavist revolution and the justice system», Latin American Policy, 8 (2) DOI: https://doi.org/10.1111/ lamp.12126.

Pérez Perdomo, Rogelio (2001). "Oil lawyers and the globalization of Venezuelan oil industry». Rogelio Pérez Perdomo (coord.), Rules and networks: The legal culture of global legal transactions (pp. 301-324). Oxford: Hart.

-. (2003). "Venezuela 1958-1999: The legal system in an impaired democracy». Lawrence Friedman y Rogelio Pérez Perdomo (eds.), Legal cultures in the age of globalization (Latin Europe and Latin America) (pp. 414-478). Stanford: Stanford University Press.

-. (2006). «La educación jurídica en Venezuela 1960-2005». Bogotá: Universidad Externado de Colombia.

-. (2011). Justicia e injusticias en Venezuela. Caracas: Academia Nacional de la Historia y Universidad Metropolitana.

-. (2016). «Reformar la educación jurídica, ¿tarea para Sísifo?». Revista Pedagogía Universitaria y Didáctica del Derecho, 3(1): 3-27.

Pinilla-Rodríguez, Diego, Héctor Reinoso-Vásquez y María Eugenia López-Merino (2017). «Complejidad, formación jurídica y transformación social. algunas anotaciones». Revista Jurídicas, 14(1): 87-101.

\section{Agradecimientos}

La investigación que soporta este estudio fue auspiciada por la Dirección de Investigaciones y la Facultad de Estudios Jurídicos y Políticos de la Universidad Metropolitana. Los autores agradecen a la profesora Mirian Rodríguez, secretaria general de la Universidad y antigua directora de la Escuela de Derecho, por los datos suministrados y por sus comentarios. Asimismo, agradecen profusamente a la estudiante de Derecho, Irene Fernández, quien fungió como asistente de investigación y cuya ayuda resultó invaluable para la realización de este proyecto.

\section{Sobre los autores}

Victoria Capriles es abogada, magíster en Sociología Jurídica del Instituto Internacional de Sociología Jurídica (Oñati, España) y actualmente se encuentra realizando estudios doctorales en ciencia política en la Universidad Simón Bolívar (Caracas, Venezuela). Se desempeña como profesora del Departamento de Estudios Internacionales y directora adjunta del Centro de Derechos Humanos de la Universidad 
Metropolitana (Caracas, Venezuela).Su correo electrónico es vcapriles@unimet.edu. ve. (D) https://orcid.org/00oo-0002-7269-1168.

Rogelio Pérez-Perdomo es abogado, magíster de leyes de la Universidad de Harvard (Cambridge, Estados Unidos) y doctor en Derecho de la Universidad Central de Venezuela (Caracas, Venezuela). Se desempeña como profesor del Departamento de Estudios Jurídicos y es exdecano de la Facultad de Estudios Políticos y Jurídicos de la Universidad Metropolitana. Individuo de número de la Academia Nacional de la Historia de Venezuela. Su correo electrónico es rperez@unimet.edu.ve. (DD https:// orcid.org/0000-0003-0086-0855. 
La Revista Pedagogía Universitaria y Didáctica del Derecho (RPUDD) es una publicación científica semestral que contribuye a la reflexión multidisciplinaria sobre pedagogía universitaria y didáctica del derecho, para la formación y consolidación de esta área de investigación; así como a la difusión de prácticas innovadoras en la enseñanza-aprendizaje del derecho considerando el contexto nacional e internacional. Es una publicación electrónica internacional con una codirección entre Brasil y Chile.

\author{
DIRECTORA \\ María Francisca Elgueta Rozas \\ Universidad de Chile \\ DIRECTOR \\ Renato Duro Dias \\ Universidad Federal de Rio Grande, Brasil \\ SITIO WEB \\ pedagogiaderecho.uchile.cl \\ CORREO ELECTRÓNICO \\ rpedagogia@derecho.uchile.cl \\ LICENCIA DE ESTE ARTÍCULO
}

Creative Commons Atribución Compartir Igual 4.o Internacional

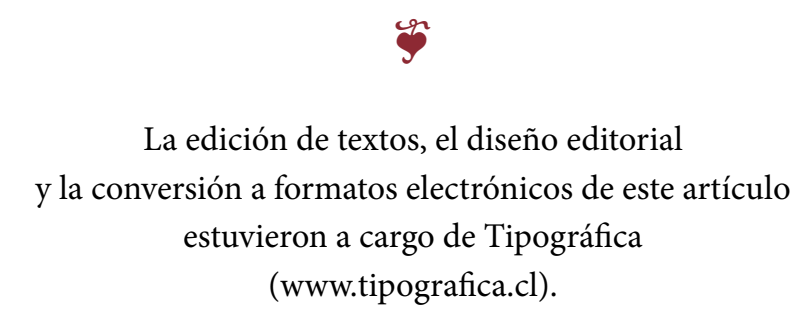

